\title{
HARP-CDP hadroproduction data: comparison with FLUKA and GEANT4 simulations
}

\author{
A. Bolshakova ${ }^{1}$, I. Boyko ${ }^{1}$, G. Chelkov ${ }^{1, b}$, D. Dedovitch ${ }^{1}$, A. Elagin ${ }^{1,2}$, D. Emelyanov ${ }^{1}$, M. Gostkin ${ }^{1}$, A. Guskov ${ }^{1}$, \\ Z. Kroumchtein ${ }^{1}$, Yu. Nefedov ${ }^{1}$, K. Nikolaev ${ }^{1}$, A. Zhemchugov ${ }^{1}$, F. Dydak ${ }^{3}$, J. Wotschack ${ }^{3, a}$, A. De Min $^{4, c}$, \\ V. Ammosov ${ }^{5, d}$, V. Gapienko ${ }^{5}$, V. Koreshev ${ }^{5}$, A. Semak ${ }^{5}$, Yu. Sviridov ${ }^{5}$, E. Usenko ${ }^{5,6}$, V. Zaets ${ }^{5}$ \\ ${ }^{1}$ Joint Institute for Nuclear Research, Dubna, Russia \\ ${ }^{2}$ Present address: Texas A\&M University, College Station, USA \\ ${ }^{3}$ CERN, Geneva, Switzerland \\ ${ }_{5}^{4}$ Politecnico di Milano and INFN, Sezione di Milano-Bicocca, Milan, Italy \\ ${ }_{6}^{5}$ Institute of High Energy Physics, Protvino, Russia \\ ${ }^{6}$ Present address: Institute for Nuclear Research RAS, Moscow, Russia
}

Received: 9 June 2010 / Revised: 17 September 2010 / Published online: 13 November 2010

(C) The Author(s) 2010. This article is published with open access at Springerlink.com

\begin{abstract}
We report on the comparison of production characteristics of secondary protons and charged pions in the interactions of protons and charged pions with momentum between $3 \mathrm{GeV} / \mathrm{c}$ and $15 \mathrm{GeV} / \mathrm{c}$ with beryllium, copper, and tantalum nuclei, with simulations by the FLUKA program and the Geant4 tool kit. Overall production cross-sections are reasonably well reproduced, within factors of two. In more detail, there are areas with poor agreement that are unsatisfactory and call for modelling improvements. Overall, the current FLUKA simulation fares better than the current Geant4 simulation.
\end{abstract}

\section{Introduction}

The HARP experiment arose from the realization that the inclusive differential cross-sections of hadron production in the interactions of few $\mathrm{GeV} / \mathrm{c}$ protons with nuclei were known only within a factor of two to three, while more precise cross-sections are in demand for several reasons. Among them are the understanding of the underlying physics and the modelling of Monte Carlo generators of hadron-nucleus collisions.

\footnotetext{
a e-mail: joerg.wotschack@cern.ch

${ }^{b}$ Also at the Moscow Institute of Physics and Technology, Moscow, Russia.

${ }^{\mathrm{c}}$ On leave of absence at Ecole Polytechnique Fédérale, Lausanne, Switzerland.

${ }^{\mathrm{d}}$ Deceased on 11 January 2010.
}

The HARP experiment was designed to carry out a programme of systematic and precise (i.e., at the few per cent level) measurements of hadron production by protons and pions with momenta from $1.5 \mathrm{GeV} / \mathrm{c}$ to $15 \mathrm{GeV} / \mathrm{c}$, on a variety of target nuclei ranging from hydrogen to lead.

The HARP detector combined a forward spectrometer with a large-angle spectrometer. The latter comprised a cylindrical Time Projection Chamber (TPC) around the target and an array of Resistive Plate Chambers (RPCs) that surrounded the TPC. The purpose of the TPC was track reconstruction and particle identification by $\mathrm{d} E / \mathrm{d} x$. The purpose of the RPCs was to complement the particle identification by time of flight.

The HARP experiment took data at the CERN Proton Synchrotron in 2001 and 2002.

Several papers reported on the measurement of inclusive cross-sections of large-angle production (polar angle $\theta$ in the range $20^{\circ}<\theta<125^{\circ}$ ) of secondary protons and charged pions, in the interactions with $5 \% \lambda_{\text {int }}$ beryllium, copper, tantalum, and lead targets, of protons and pions with beam momenta of $\pm 3.0, \pm 5.0, \pm 8.0$ ( +8.9 for beryllium), \pm 12.0 , and $\pm 15.0 \mathrm{GeV} / \mathrm{c}$ [1-5]. Besides, one paper [6] reported on disagreements between data and simulations by the Geant 4 tool kit $[7,8]$, which led to significant improvements in its simulation code [9].

In this paper, we report in more detail on comparisons of hadroproduction data with simulations by the FLUKA program $[10,11]$ and the Geant4 tool kit.

Our work involves only the HARP large-angle spectrometer. The data analysis is based on our calibrations of the HARP TPC and RPCs published in [12, 13]. 
Fig. 1 Comparison of measured inclusive $\pi^{+}$ production cross-sections by protons on $\mathrm{Be}$ (squares), $\mathrm{Cu}$ (triangles) and Ta (circles) with Geant4 simulations employing the QGSP_BERT (upper left panel), QGSP_BIC (upper right panel), QGSP_EMV (lower left panel) and FTFP_BERT (lower right panel), in the

intermediate-angle region, as a function of beam momentum; the data are shown with black symbols, the simulations with open symbols; here and in further similar figures, the simulated points are connected by lines to guide the eye
HARP-CDP pA $\rightarrow \pi^{+} X 20^{\circ}<\Theta<50^{\circ}$

HARP-CDP pA $\rightarrow \pi^{+} X \quad 20^{\circ}<\Theta<50^{\circ}$
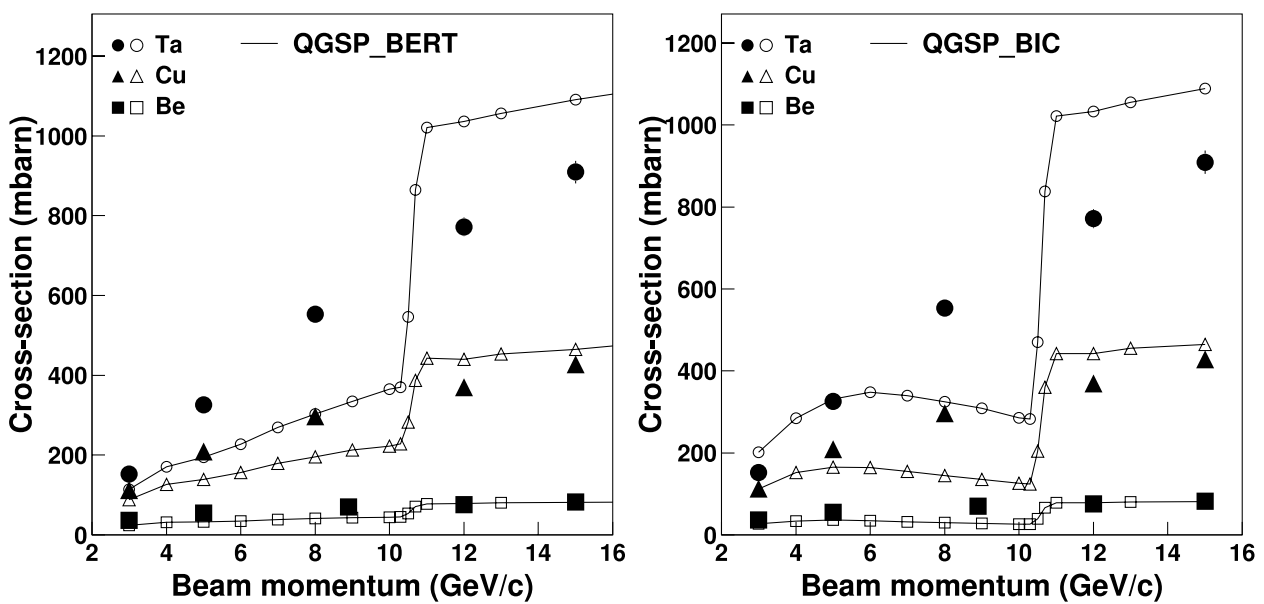

HARP-CDP pA $\rightarrow \pi^{+} X 20^{\circ}<\Theta<50^{\circ}$
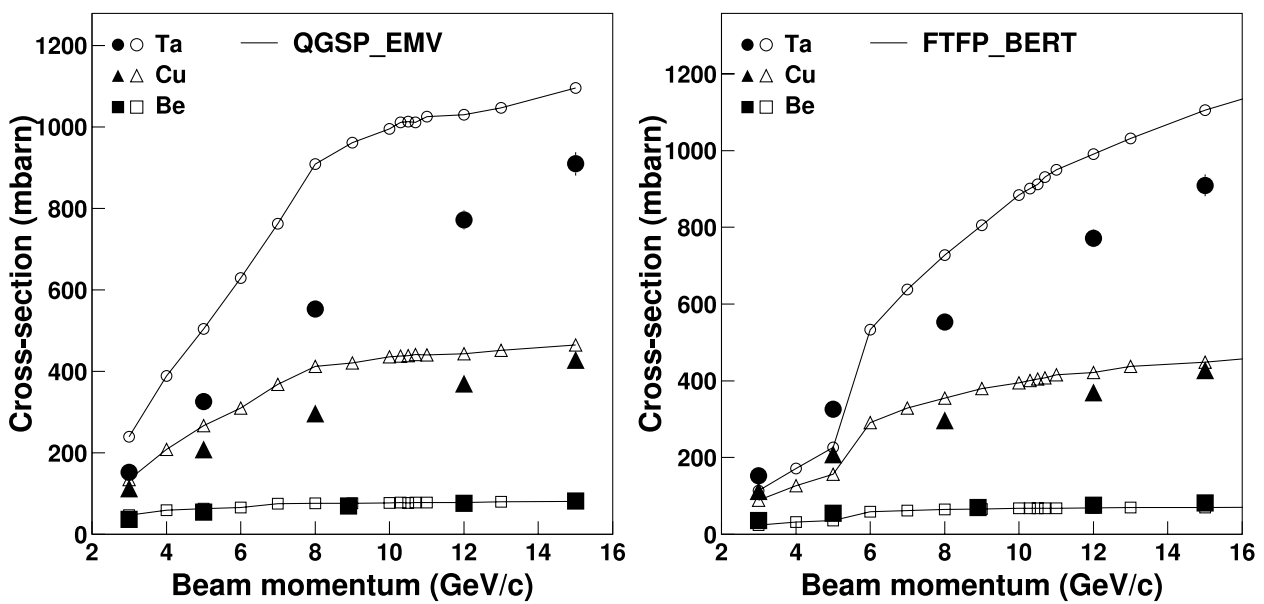

\section{The beams and the HARP spectrometer}

The protons and pions were delivered by the T9 beam line in the East Hall of CERN's Proton Synchrotron. This beam line supports beam momenta between $1.5 \mathrm{GeV} / \mathrm{c}$ and $15 \mathrm{GeV} / \mathrm{c}$, with a momentum bite $\Delta p / p \sim 1 \%$.

The beam instrumentation, the definition of the beam particle trajectory, the cuts to select 'good' beam particles, and the muon and electron contaminations of the particle beams, are the same as described, e.g., in [4]. The targets were discs made of high-purity material, $5 \% \lambda_{\text {int }}$ thick.

The momentum resolution $\sigma\left(1 / p_{\mathrm{T}}\right)$ of the HARP-TPC is typically $0.2(\mathrm{GeV} / \mathrm{c})^{-1}$ and worsens towards small relative particle velocity $\beta$ and small polar angle $\theta$. The absolute momentum scale is determined to be correct to better than $2 \%$, both for positively and negatively charged particles.
The polar angle $\theta$ is measured in the TPC with a resolution of $\sim 13 \mathrm{mrad}$, for a proton with $p_{\mathrm{T}}=500 \mathrm{MeV} / \mathrm{c}$ in the TPC gas and a polar angle of $\theta=60^{\circ}$. The polar-angle scale is correct to better than 2 mrad.

The TPC measures $\mathrm{d} E / \mathrm{d} x$ with a resolution of $16 \%$ for a track length of $300 \mathrm{~mm}$.

The intrinsic efficiency of the RPCs that surround the TPC is better than $98 \%$.

The intrinsic time resolution of the RPCs is $127 \mathrm{ps}$ and the system time-of-flight resolution (that includes the jitter of the arrival time of the beam particle at the target) is $175 \mathrm{ps}$.

To separate measured particles into species, we assign on the basis of $\mathrm{d} E / \mathrm{d} x$ and $\beta$ to each particle a probability of being a proton, a pion (muon), or an electron, respectively. The probabilities add up to unity, so that the number of particles is conserved. These probabilities are used for weighting when entering tracks into plots or tables. 

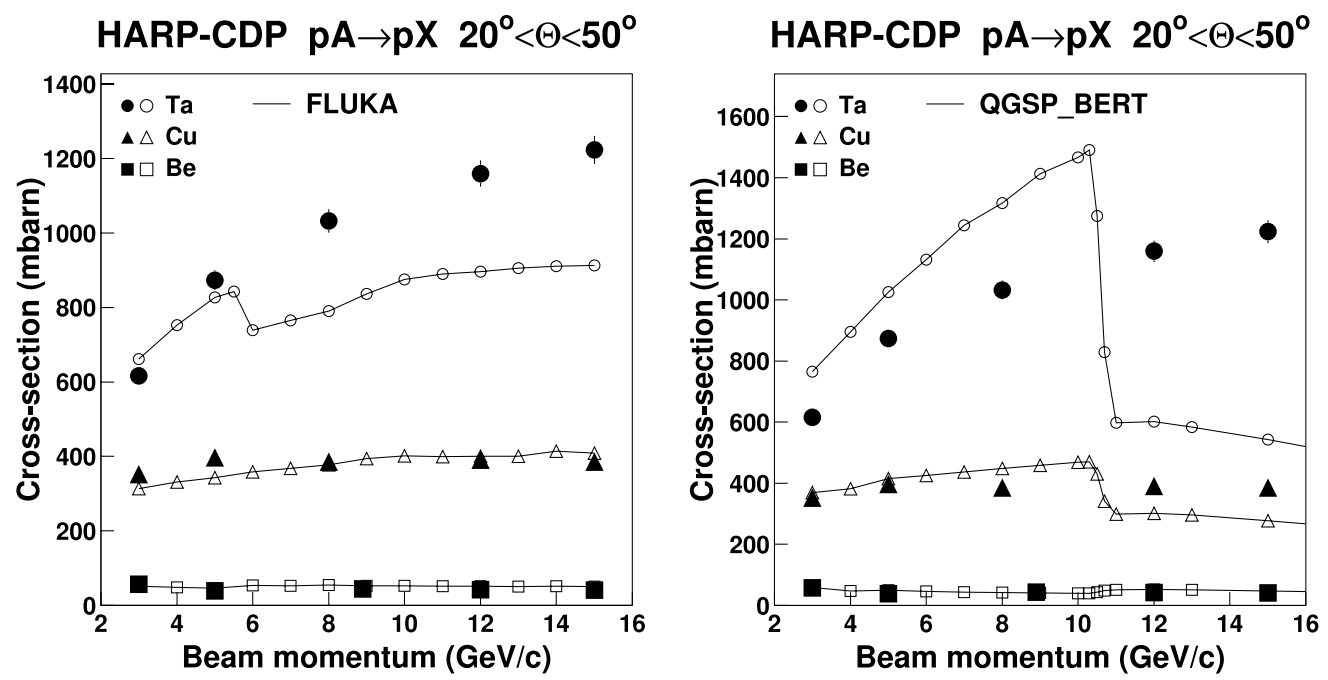

Fig. 2 Comparison of measured (black symbols) inclusive proton production cross-sections by protons on $\mathrm{Be}, \mathrm{Cu}$ and Ta nuclei, in the intermediate-angle region, as a function of beam momentum, with FLUKA (left panel) and Geant4 (right panel) simulations (open symbols)

HARP-CDP $\mathrm{pA} \rightarrow \pi^{+} X 20^{\circ}<\Theta<50^{\circ}$

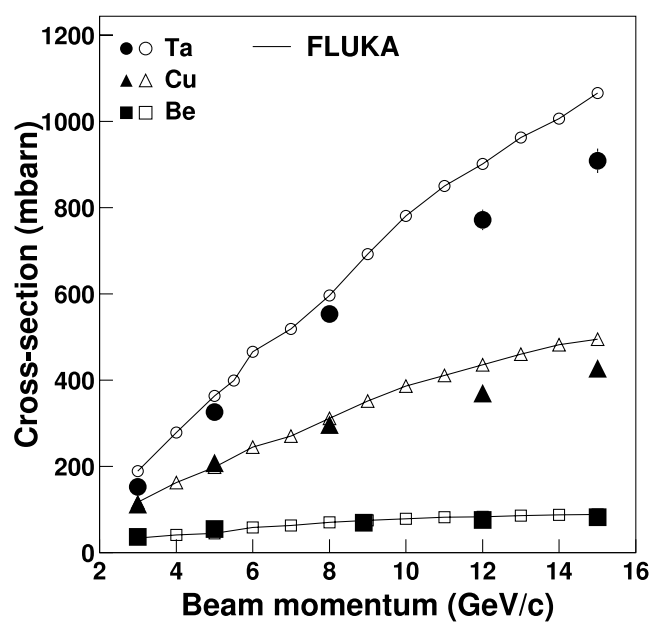

HARP-CDP PA $\rightarrow \pi^{+} X 20^{\circ}<\Theta<50^{\circ}$

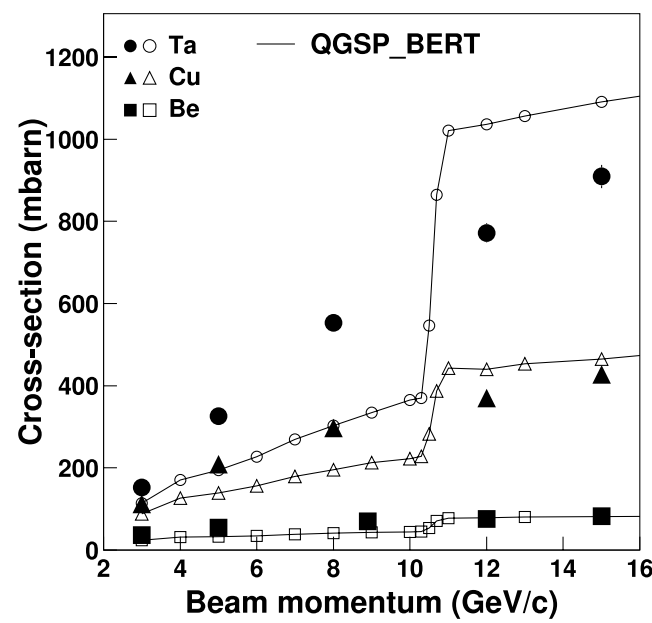

HARP-CDP pA $\rightarrow \pi^{-} X 20^{\circ}<\Theta<50^{\circ}$

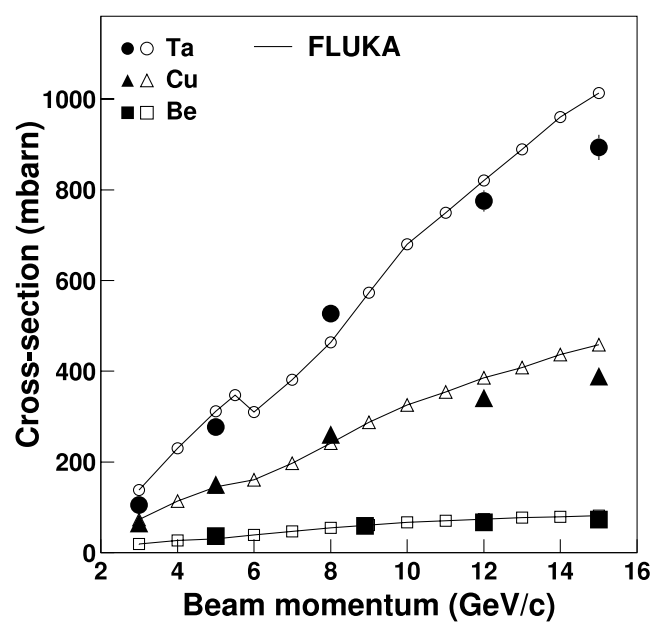

HARP-CDP $\mathrm{pA} \rightarrow \pi^{-} X 20^{\circ}<\Theta<50^{\circ}$

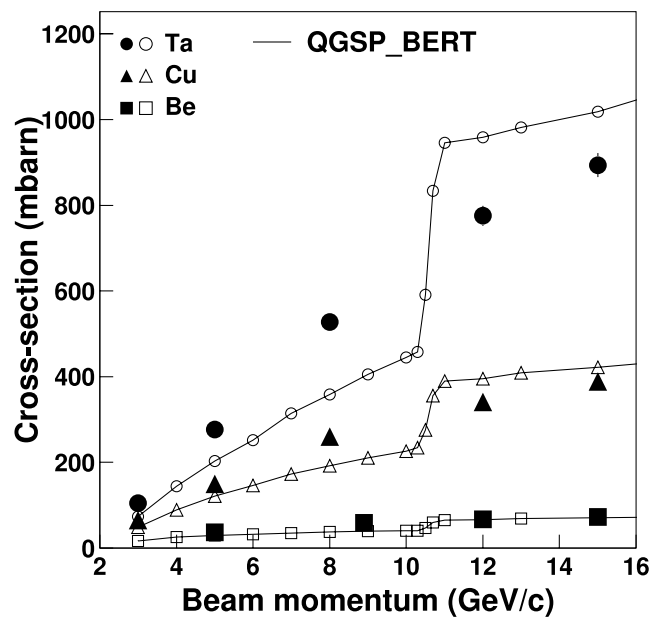

Fig. 3 Comparison of measured inclusive $\pi^{+}$(left panels) and $\pi^{-}$(right panels) production cross-sections by protons on Be, Cu and Ta nuclei (black symbols), in the intermediate-angle region, with FLUKA and Geant4 simulations (open symbols) 


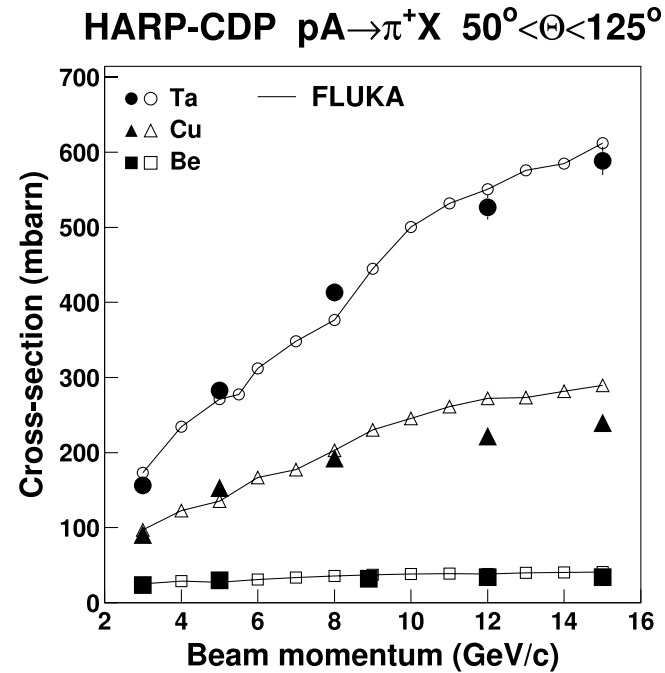

HARP-CDP pA $\rightarrow \pi^{-} \times \quad 50^{\circ}<\Theta<125^{\circ}$

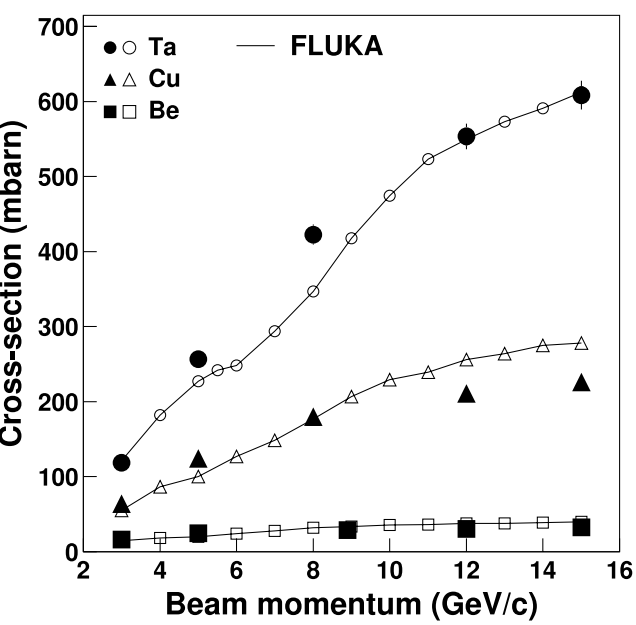

HARP-CDP pA $\rightarrow \pi^{+} X \quad 50^{\circ}<\Theta<125^{\circ}$

HARP-CDP pA $\rightarrow \pi^{-} X 50^{\circ}<\Theta<125^{\circ}$
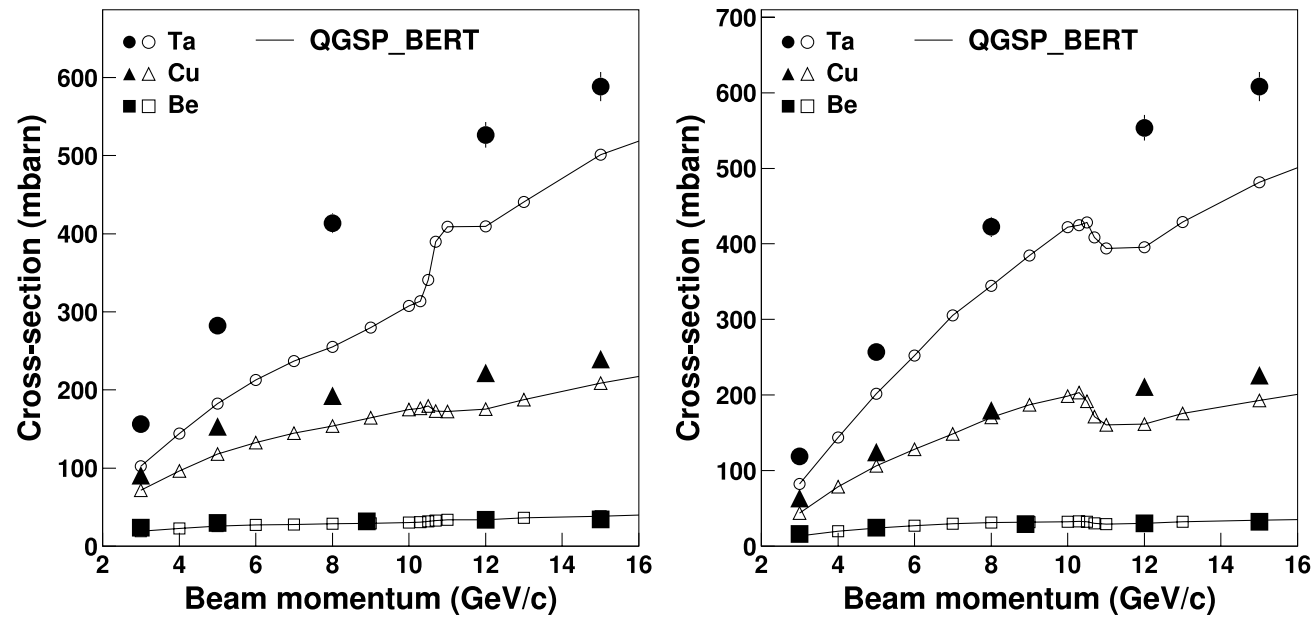

Fig. 4 Same as Fig. 3 but in the large-angle region
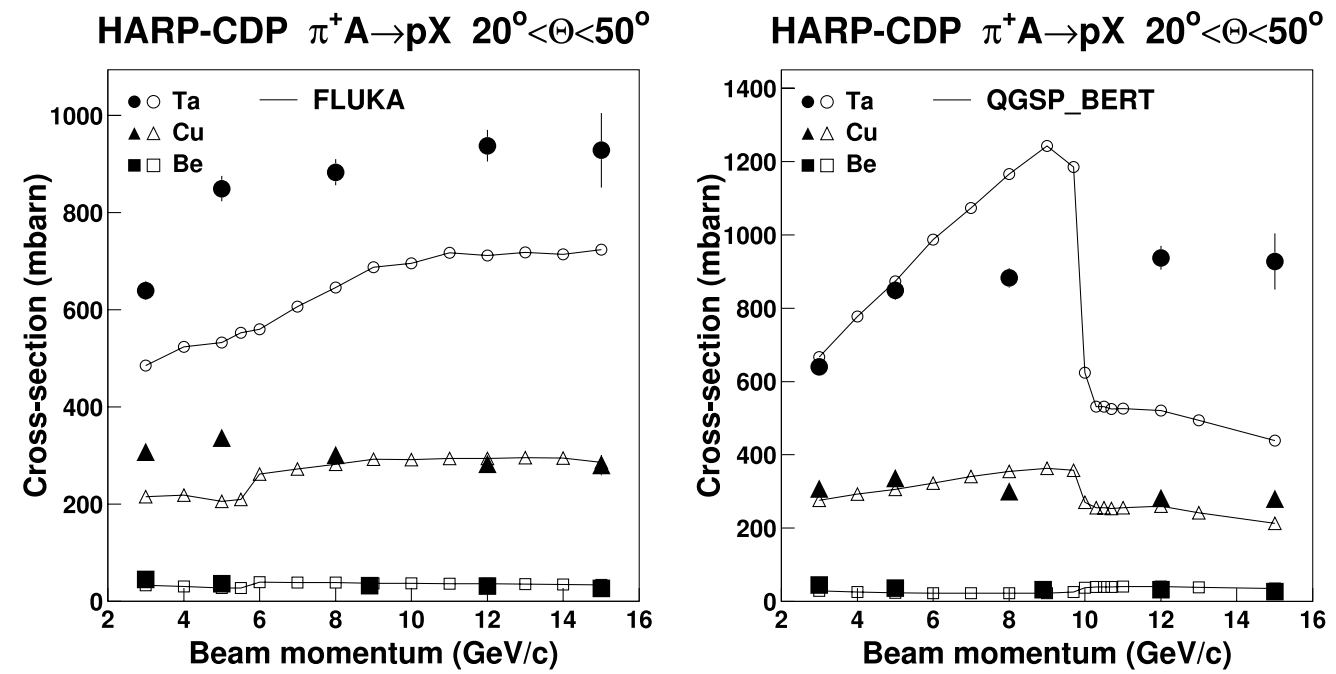

Fig. 5 Comparison of measured (black symbols) inclusive proton production cross-sections by $\pi^{+}$'s on Be, $\mathrm{Cu}$ and Ta nuclei, in the intermediate-angle region, as a function of beam momentum, with FLUKA (left panel) and Geant4 (right panel) simulations (open symbols) 
Fig. 6 Comparison of measured inclusive $\pi^{+}$(left panels) and $\pi^{-}$(right panels) production cross-sections by $\pi^{+}$'s on $\mathrm{Be}, \mathrm{Cu}$ and Ta nuclei (black symbols), in the intermediate-angle region, with FLUKA and Geant4 simulations (open symbols)
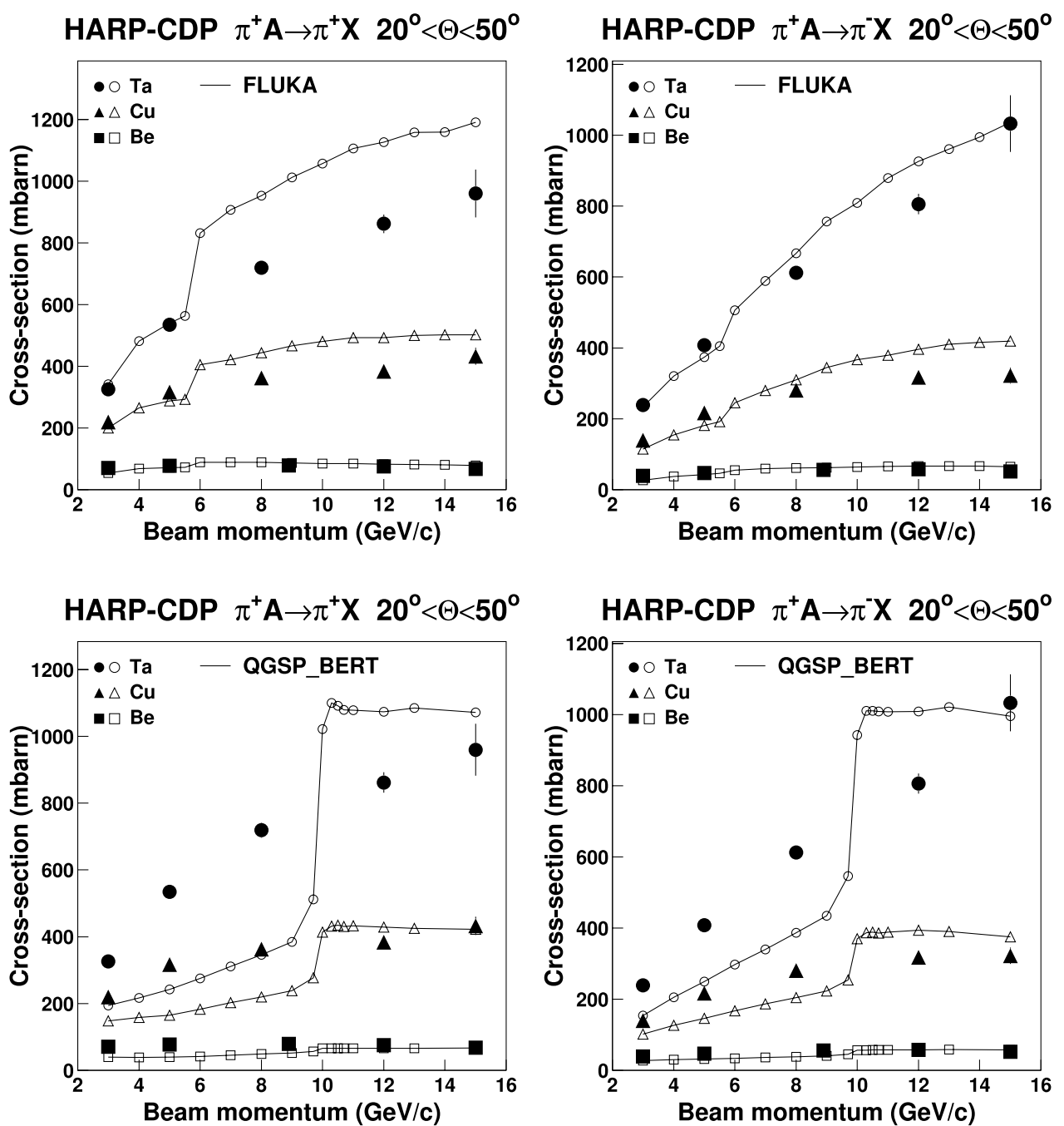

\section{Modus operandi of the comparison between data and simulations}

All data shown in this paper have been published [1-5] in the form of double-differential inclusive cross-sections $\mathrm{d}^{2} \sigma / \mathrm{d} p \mathrm{~d} \Omega[\mathrm{mbarn} /(\mathrm{sr} \cdot \mathrm{GeV} / \mathrm{c})]$, in the transverse-momentum range $0.10<p_{\mathrm{T}}<1.25 \mathrm{GeV} / \mathrm{c}$ and the polar-angle range $20^{\circ}<\theta<125^{\circ}$. For ease of use, the cross-sections are also available in computer-readable form as ASCII files [14-18].

For the comparison with simulations, cross-sections are integrated over two regions: the 'intermediate-angle' region $\left(20^{\circ}<\theta<50^{\circ}\right)$ and the 'large-angle' region $\left(50^{\circ}<\right.$ $\theta<125^{\circ}$ ). For $\pi^{+}$and $\pi^{-}$secondaries, the $p_{\mathrm{T}}$ ranges are $0.10<p_{\mathrm{T}}<0.72 \mathrm{GeV} / \mathrm{c}$ in the intermediate-angle region, and $0.16<p_{\mathrm{T}}<1.25 \mathrm{GeV} / \mathrm{c}$ in the large-angle region. For proton secondaries, the $p_{\mathrm{T}}$ range is $0.30<p_{\mathrm{T}}<$ $0.72 \mathrm{GeV} / \mathrm{c}$, for the large energy loss by ionization in the target and in materials before entering the active TPC volume.
Cross-sections of protons are given only in the intermediateangle region because the minimum $p_{\mathrm{T}}$ of protons in the large-angle region is even larger, about twice the one in the intermediate-angle region.

The measured inclusive cross-sections in the said regions were obtained by integrating over respective bins of polar angle and transverse momentum, taking into account the correlation of systematic errors. The uncertainties of the shown inclusive cross-sections are at the level of 3\%. The contribution of statistical errors is negligible, with the exception of the cross-sections for $15 \mathrm{GeV} / \mathrm{c} \pi^{+}$beams. The largest contributions to the systematic error arise from the overall normalization (2\%) and from the uncertainty of the momentum scale of secondaries (2\%).

The Monte Carlo programs FLUKA and Geant 4 are run with protons and charged-pion beams with the same beam momenta that interact with beryllium, copper and tantalum target nuclei. Only final-state hadrons that stem from the target nuclei are taken into account. For the same regions se- 
HARP-CDP $\pi^{+} A \rightarrow \pi^{+} X \quad 50^{\circ}<\Theta<125^{\circ}$

HARP-CDP $\pi^{+} A \rightarrow \pi^{-} X 50^{\circ}<\Theta<125^{\circ}$
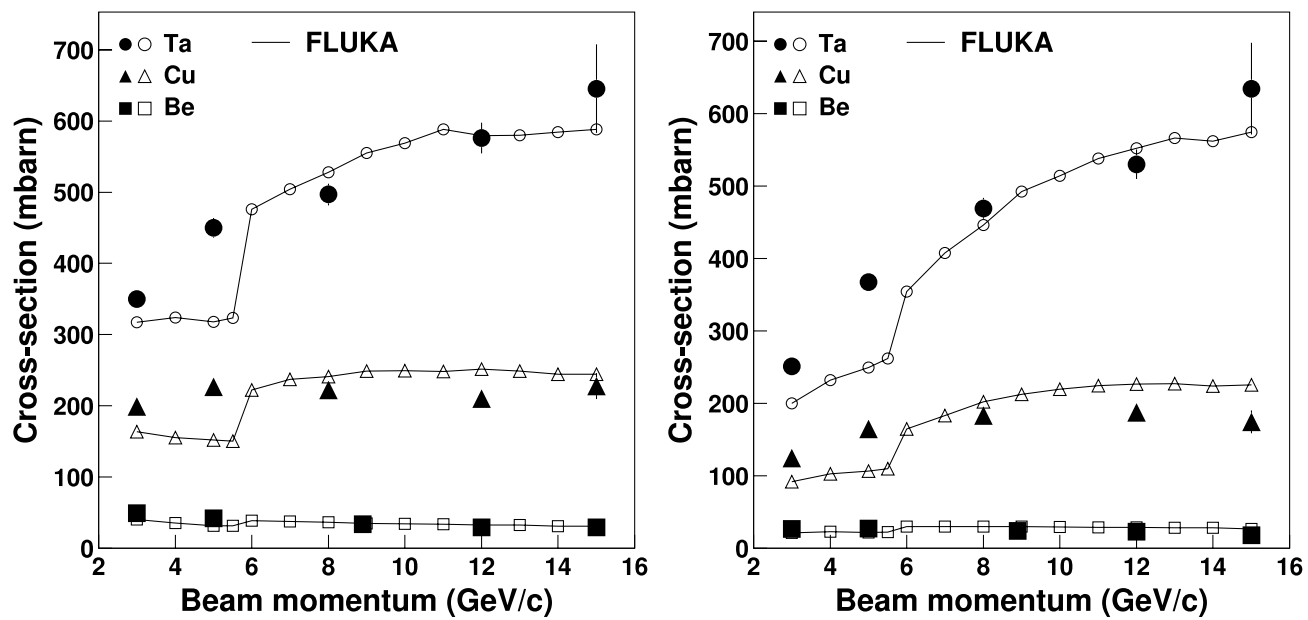

HARP-CDP $\pi^{+} A \rightarrow \pi^{+} X \quad 50^{\circ}<\Theta<125^{\circ}$

HARP-CDP $\pi^{+} A \rightarrow \pi^{-} X 50^{\circ}<\Theta<125^{\circ}$
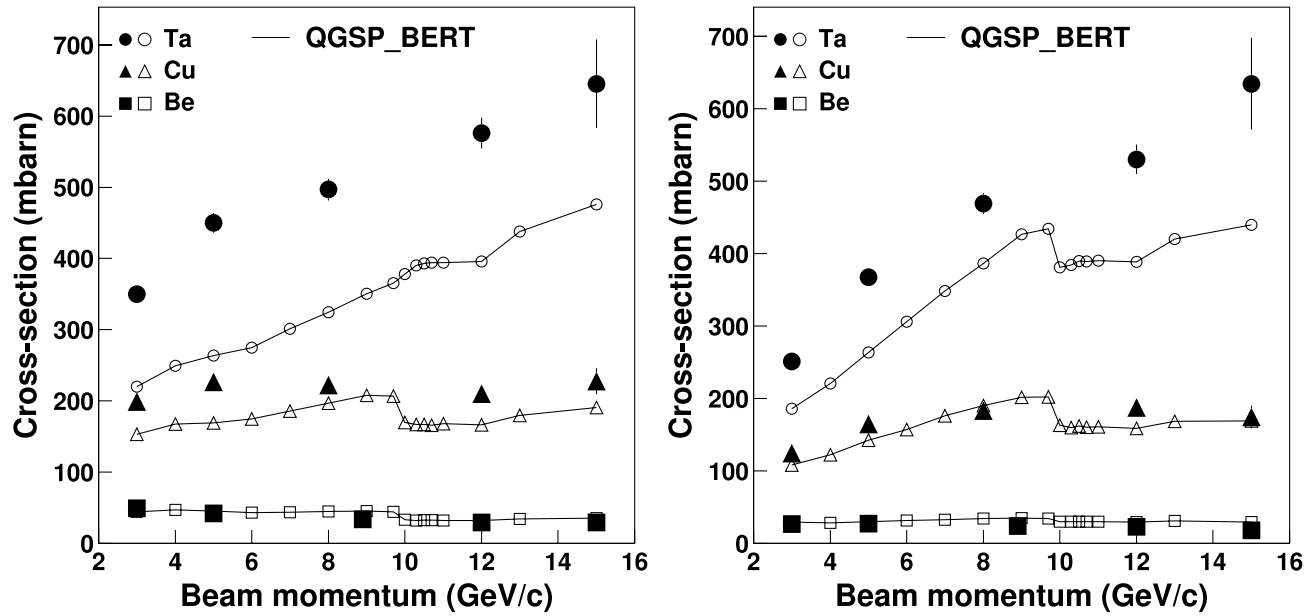

Fig. 7 Same as Fig. 6 but in the large-angle region
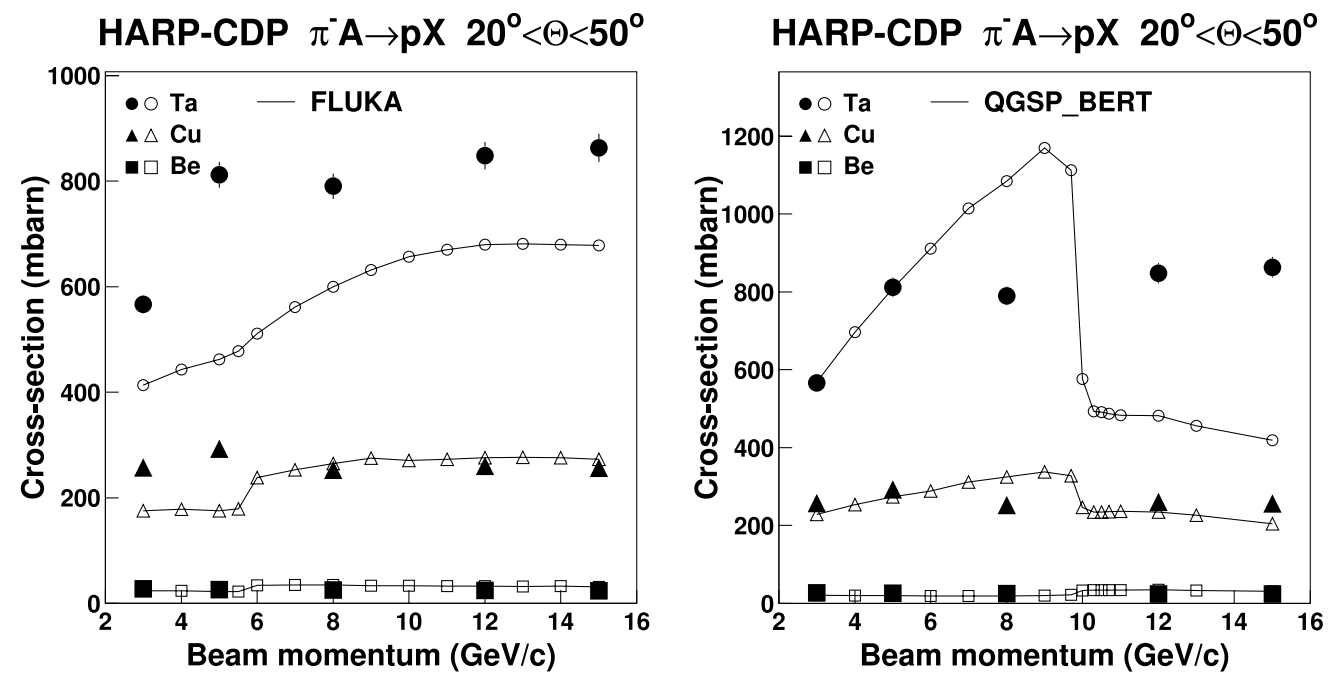

Fig. 8 Comparison of measured (black symbols) inclusive proton production cross-sections by $\pi^{- \text {'s }}$ on $\mathrm{Be}$, $\mathrm{Cu}$ and Ta nuclei, in the intermediate-angle region, as a function of beam momentum, with FLUKA (left panel) and Geant4 (right panel) simulations (open symbols) 
Fig. 9 Comparison of measured inclusive $\pi^{+}$(left panels) and $\pi^{-}$(right panels) production cross-sections by $\pi^{-}$'s on $\mathrm{Be}, \mathrm{Cu}$ and Ta nuclei (black symbols), in the intermediate-angle region, with FLUKA and Geant4 simulations (open symbols)

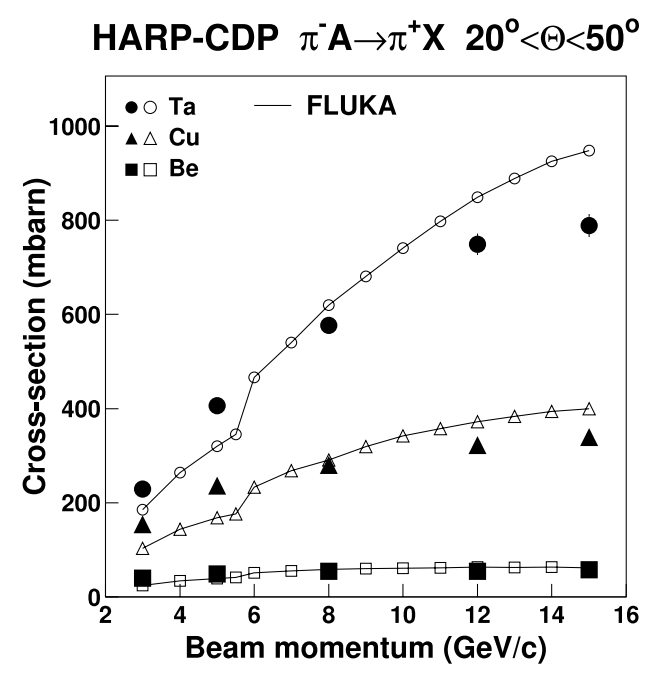

HARP-CDP $\pi^{-} A \rightarrow \pi^{-} X \quad 20^{\circ}<\Theta<50^{\circ}$
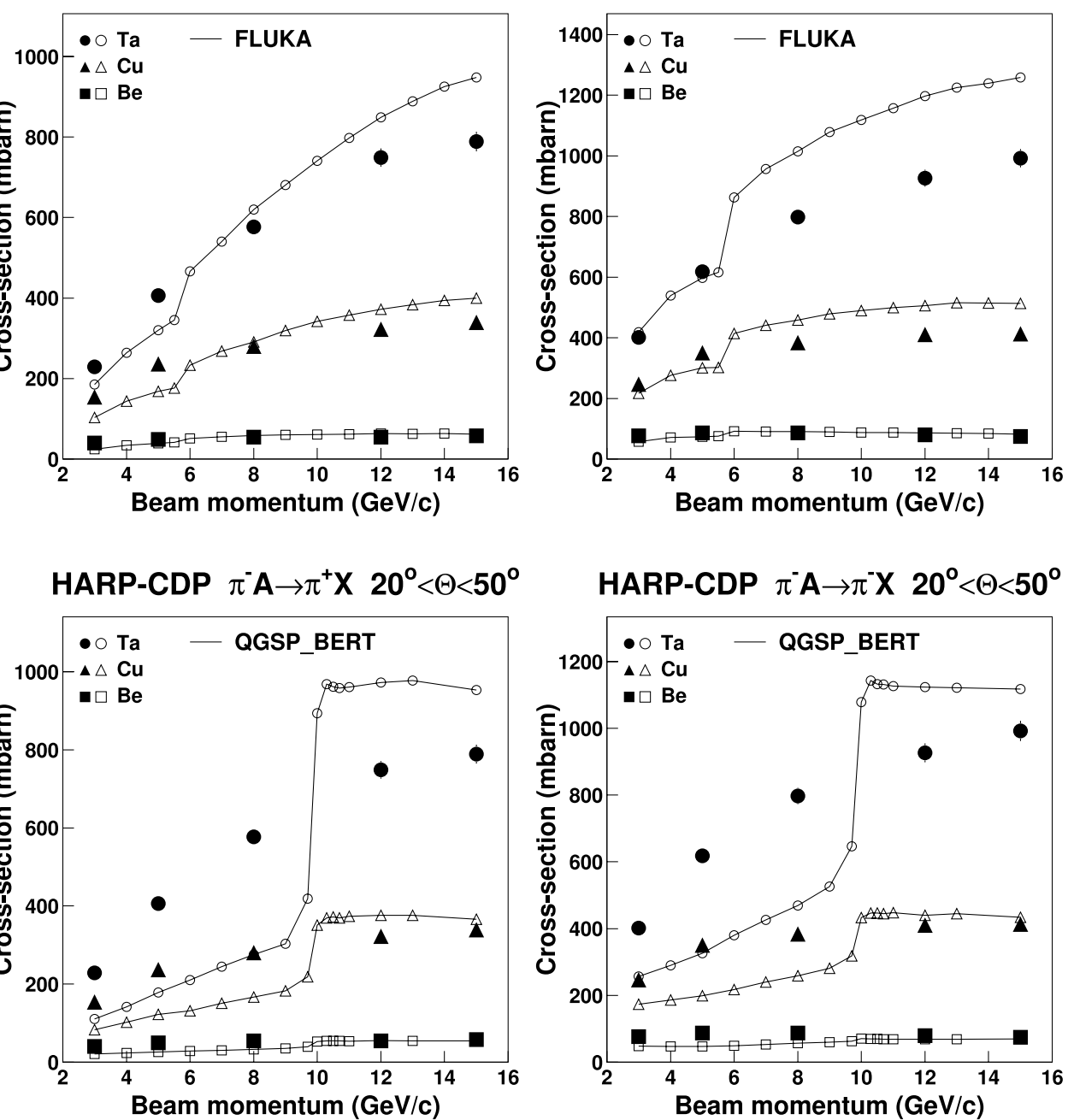

lected for the presentation of data, integrated inclusive crosssections are extracted.

The comparison is made separately (i) for the intermediate-angle and the large-angle regions, (ii) for secondary

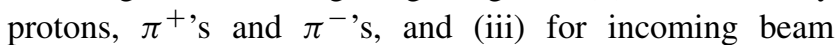
protons, $\pi^{+}$'s and $\pi^{-}$'s in the momentum range $3-15 \mathrm{GeV} / \mathrm{c}$.

We used FLUKA 2008.3c in its default configuration. We did not make use of the option to extend FLUKA's PEANUT model to projectile momenta above $5 \mathrm{GeV} / \mathrm{c}$ which is claimed by the FLUKA authors to lead to improvements.

For Geant4, we used version 4.9.3. The QGSP_BERT 'physics list' was selected for being the preferred choice of the LHC Collaborations ATLAS and CMS [19]. In order to assess the differences with other popular physics lists, in Fig. 1 QGSP_BERT simulations of inclusive $\pi^{+}$ production by protons on beryllium, copper and tanta- lum nuclei are compared with the simulations employing the Geant4 physics lists QGSP_BIC, QGSP_EMV and FTFP_BERT. There are remarkable differences between the simulations, similar in size as the differences between the data and, e.g., the QGSP_BERT simulation. Most of these differences show up for beam proton momenta below $10 \mathrm{GeV} / \mathrm{c}$. Between 10 and $15 \mathrm{GeV} / \mathrm{c}$ beam proton momentum, the chosen four physics lists give comparable results.

Our aim is not to test all possible Geant 4 physics lists against our data. Rather, we wish to point to areas, using the QGSP_BERT physics list as an example, where data and simulation seriously disagree.

We note that the beta version of Geant 4.9.4 is claimed by the Geant 4 authors to lead to improvements.

We trust that our data are useful to the simulation developers and provide guidance for modelling improvements. 
Fig. 10 Same as Fig. 9 but in the large-angle region

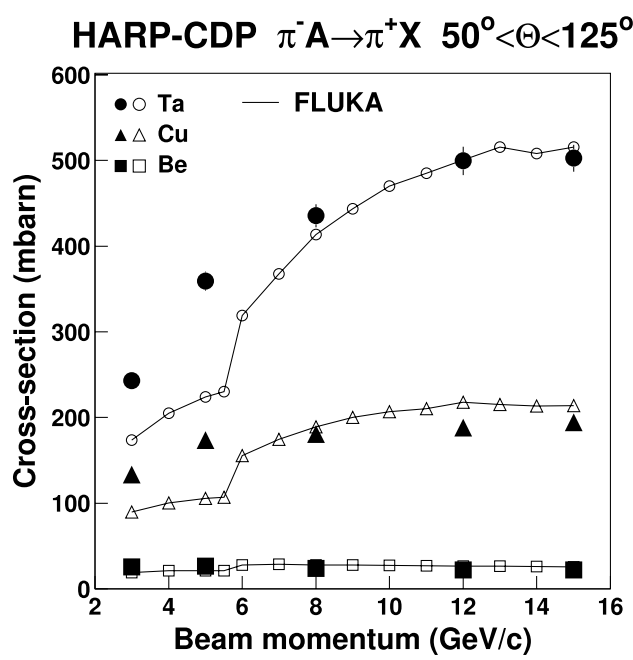

HARP-CDP $\pi^{-} A \rightarrow \pi^{-} X \quad 50^{\circ}<\Theta<125^{\circ}$

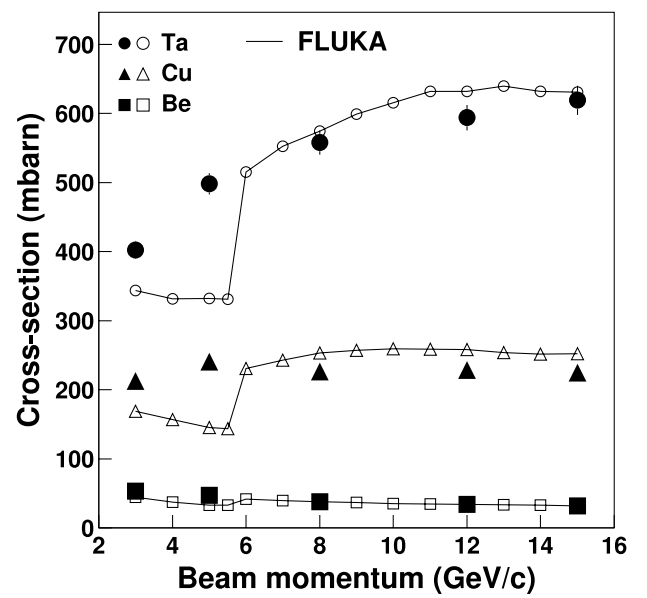

HARP-CDP $\pi^{-} A \rightarrow \pi^{+} X \quad 50^{\circ}<\Theta<125^{\circ}$

HARP-CDP $\pi^{-} \mathrm{A} \rightarrow \pi^{-} \mathrm{X} \quad 50^{\circ}<\Theta<125^{\circ}$

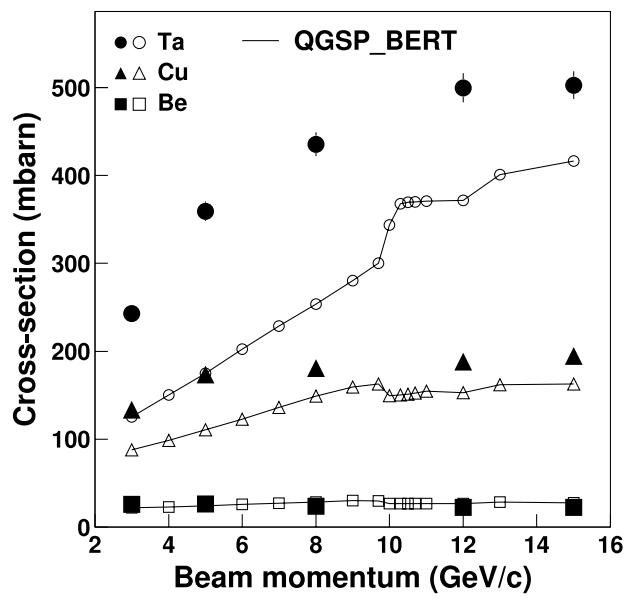

The comparison of the data with FLUKA and Geant4 QGSP_BERT simulations is concentrated in Sect. 4. A critical appraisal is found in Sect. 6.

\section{Comparison of data with FLUKA and Geant4 simulations}

Figures 2-4 show comparisons with FLUKA and Geant4 simulations of measured inclusive proton, $\pi^{+}$and $\pi^{-}$production cross-sections by protons on $\mathrm{Be}, \mathrm{Cu}$ and Ta nuclei. For final-state protons, only comparisons in the intermediate-angle region are given (Fig. 2). For final-state $\pi^{+}$'s and $\pi^{-}$'s, comparisons are given in the intermediate-angle (Fig. 3) and large-angle regions (Fig. 4).

While Figs. 2-4 show comparisons for proton beam particles, Figs. 5, 6, 7 and Figs. 8, 9, 10 show the same comparisons for $\pi^{+}$and $\pi^{-}$beam particles, respectively.

\section{Effects from re-interactions of hadrons in nuclear matter}

Before our appraisal of the comparison of data with simulations in Sect. 6, it is useful to point to generic features of final-state hadrons from heavy nuclei.

Figure 11 shows inclusive cross-sections of proton production with polar angle $20^{\circ}<\theta<50^{\circ}$ by incoming $\pi^{-}$ beam particles with momenta in the range $3-15 \mathrm{GeV} / \mathrm{c}$, for beryllium and tantalum nuclei. The tantalum crosssections are shown as measured, while the beryllium crosssections are scaled with $\left(A_{\mathrm{Ta}} / A_{\mathrm{Be}}\right)^{0.7}$. The rationale is that the latter cross-sections are the ones on a hypothetical tantalum nucleus that has the same small re-interaction probability of secondaries as a beryllium nucleus. Figure 11 tells that the measured inclusive cross-section of tantalum is still four times larger than the scaled beryllium cross-section. Therefore, the bulk of final-state protons stem from re-interactions of secondaries in the nuclear matter of the tantalum nucleus-which after all has 


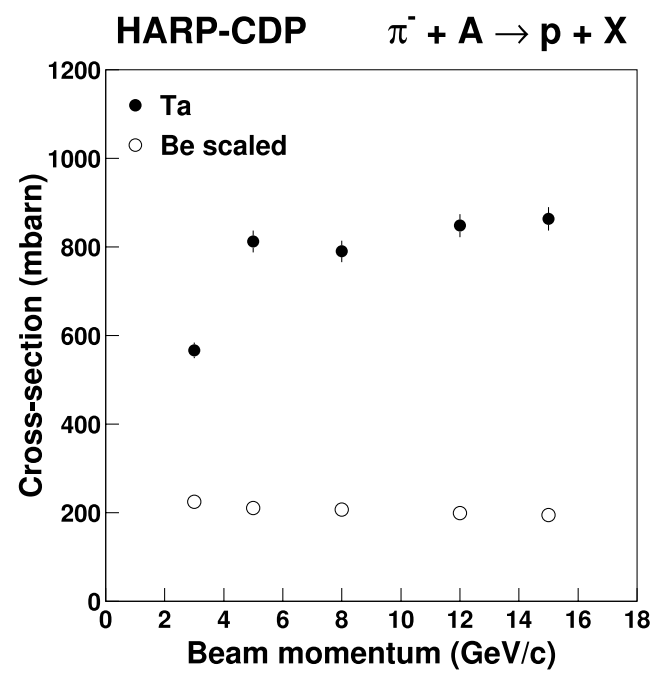

Fig. 11 Inclusive cross-sections of proton-production with polar angle $20^{\circ}<\theta<50^{\circ}$ by incoming $\pi^{-}$beam particles with momenta in the range $3-15 \mathrm{GeV} / \mathrm{c}$, for beryllium and tantalum nuclei; The tantalum cross-sections are those measured, while the beryllium cross-sections are scaled with $\left(A_{\mathrm{Ta}} / A_{\mathrm{Be}}\right)^{0.7}$

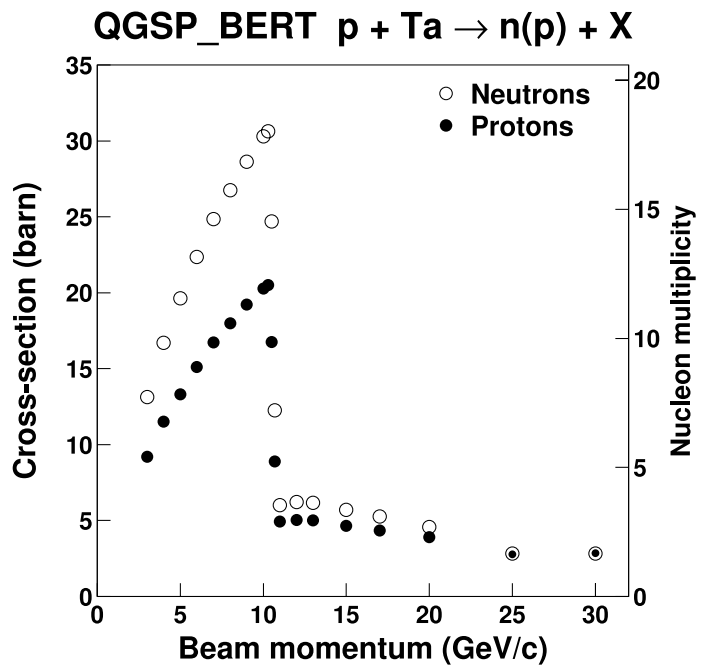

Fig. 12 Geant4 simulation (QGSP_BERT physics list) of the generation of secondary protons and neutrons with $p_{\mathrm{T}}>0.1 \mathrm{GeV} / \mathrm{c}$ in the interaction of protons with Ta nuclei

a diameter that is equivalent to several nuclear interaction lengths.

\section{Appraisal of the differences between data and simulations}

\subsection{FLUKA versus data}

In our parameter range of the comparison of data with FLUKA simulations, the latter agree with data within some $30 \%$.
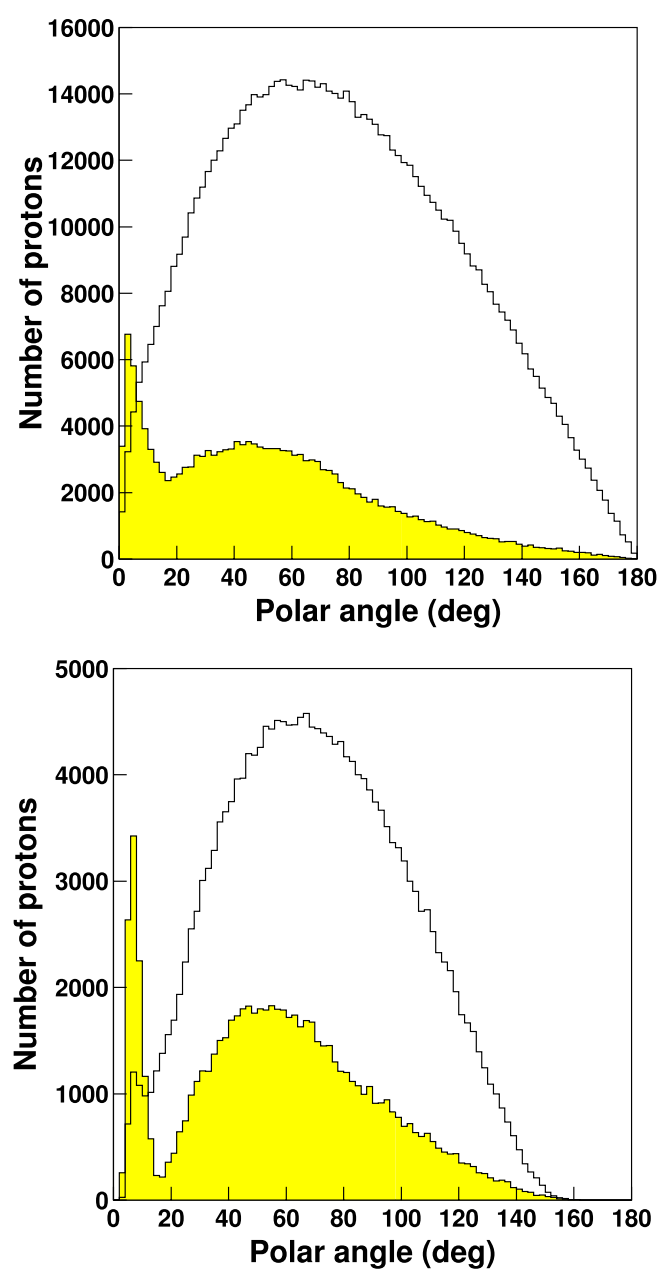

Fig. 13 (Color online) The polar-angle distribution of protons generated by Geant4 (QGSP_BERT physics list) in the interaction of protons with Ta nuclei; the open histograms refer to proton beam momenta just below the discontinuity around $10 \mathrm{GeV} / \mathrm{c}$ beam momentum, the shaded (yellow) histograms to proton beam momenta just above; the upper panel has no cut applied while the lower panel shows protons with $p_{\mathrm{T}}>0.3 \mathrm{GeV} / \mathrm{c}$

The most unsatisfactory feature in the FLUKA simulation is the discontinuity around $5 \mathrm{GeV} / \mathrm{c}$ beam momentum which is rather persistent in all combinations of beam particle and final-state particle.

\subsection{Geant4 versus data}

In our parameter range of the comparison of data with Geant4 QGSP_BERT simulations, the latter agree with data within a factor of about two.

An unacceptable feature of the Geant4 QGSP_BERT simulation is the strong discontinuity around $10 \mathrm{GeV} / \mathrm{c}$ beam momentum which is also rather persistent in all combinations of beam particle and final-state particle.

With a view to exploring further this discontinuity around $10 \mathrm{GeV} / \mathrm{c}$ in Geant4, Fig. 12 shows the simulation of the in- 

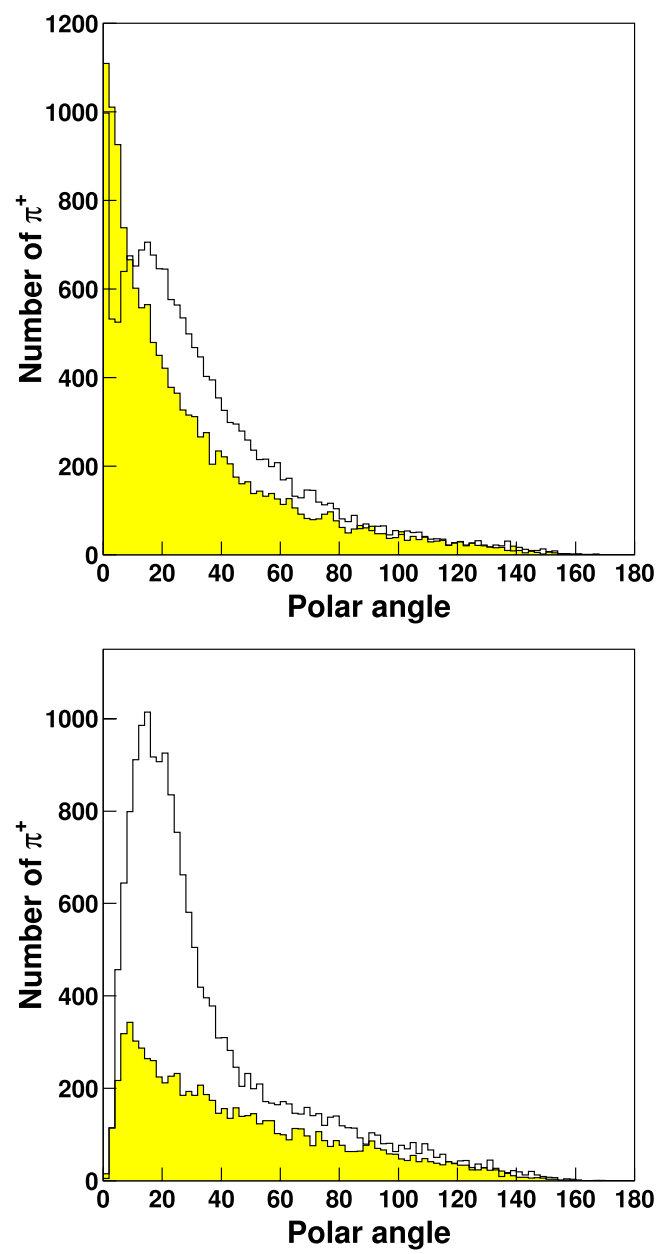

Fig. 14 (Color online) The polar-angle distributions of $\pi^{+}$'s generated by protons interacting with Ta nuclei, with no cuts applied; the upper panel shows the FLUKA simulation, the lower panel the Geant4 (QGSP_BERT physics list) simulation; the open histograms refer to proton beam momenta just above the discontinuities (around $5 \mathrm{GeV} / \mathrm{c}$ for FLUKA, around $10 \mathrm{GeV} / \mathrm{c}$ for Geant4), the shaded (yellow) histograms to proton beam momenta just below

clusive generation of secondary neutrons and protons with a rather loose cut of $p_{\mathrm{T}}>0.1 \mathrm{GeV} / \mathrm{c}$, in the interaction of beam protons with momentum in the range $3-30 \mathrm{GeV} / \mathrm{c}$ with Ta nuclei. Most prominent is an abrupt and unphysical change of modelling around $10 \mathrm{GeV} / \mathrm{c}$. Further, a comparison of the large proton multiplicity shown in Fig. 12-up to some 12 protons per beam particle interaction-with the measured much smaller proton multiplicity (see, e.g., [5]) suggests that our acceptance cut $p_{\mathrm{T}}>0.3 \mathrm{GeV} / \mathrm{c}$ favours the few protons from the interactions of the primary beam particle or of secondary hadrons in the nuclear matter of the target nucleus, but disfavours the many final-state protons from the fragmentation of the target nucleus.

Figure 13 shows the polar-angle distribution of protons generated by Geant4 (QGSP_BERT physics list), in the interaction of protons with Ta nuclei. The open histograms refer to proton beam momenta below the discontinuity around
$10 \mathrm{GeV} / \mathrm{c}$ beam momentum, the shaded (yellow) histograms to proton beam momenta above. The upper panel has no cut applied while the lower panel shows protons with $p_{\mathrm{T}}>$ $0.3 \mathrm{GeV} / \mathrm{c}$. The discrepancy between the distributions below and above the discontinuity around $10 \mathrm{GeV} / \mathrm{c}$ is unphysical.

Figure 14 shows the polar-angle distributions of $\pi^{+}$'s generated by protons interacting with Ta nuclei. No cuts are applied. The upper panel shows the FLUKA simulation, the lower panel the Geant4 (QGSP_BERT physics list) simulation. Here the open histograms refer to proton beam momenta just above the discontinuities discussed before (around $5 \mathrm{GeV} / \mathrm{c}$ for FLUKA, around $10 \mathrm{GeV} / \mathrm{c}$ for Geant4), the shaded (yellow) histograms to proton beam momenta just below. The differences of the polar-angle distributions below and above the discontinuities are unphysical.

Based on the same raw data from which we extracted our inclusive cross-sections, the HARP Collaboration published inclusive cross-sections of the production of $\pi^{+}$and $\pi^{-}$ (but not of protons) by incoming proton, $\pi^{+}$and $\pi^{-}$beam particles, in the polar-angle region $0.35 \mathrm{rad} \leq \theta \leq 2.15 \mathrm{rad}$ $[20,21]$, and compared their results with predictions of the Geant4 and MARS [22, 23] Monte Carlo programs. Substantial discrepancies were observed between their and our inclusive cross-sections, as documented in [1-5], discussed in [24-28], and summarized in the Appendix of [1].

\section{Synopsis}

A comprehensive comparison of measured inclusive crosssections of proton, $\pi^{+}$and $\pi^{-}$production by beams of protons, $\pi^{+}$'s and $\pi^{-}$'s with momentum in the range 3$15 \mathrm{GeV} / \mathrm{c}$, interacting with beryllium, copper and tantalum target nuclei, with simulations by the FLUKA program and the Geant 4 tool kit is presented. Overall production crosssections are reasonably well reproduced, within factors of two. In more detail, there are areas with poor agreement that are unsatisfactory and call for modelling improvements. Overall, the current FLUKA simulation fares better than the current Geant4 simulation.

We recall that we used FLUKA 2008.3c in its default configuration and no use was made of the option to extend FLUKA's PEANUT model to projectile momenta above $5 \mathrm{GeV} / \mathrm{c}$. For Geant 4 , the version 4.9.3 was used.

Acknowledgements We are greatly indebted to many technical collaborators whose diligent and hard work made the HARP detector a well-functioning instrument. We thank all HARP colleagues who devoted time and effort to the design and construction of the detector, to data taking, and to setting up the computing and software infrastructure. We express our sincere gratitude to HARP's funding agencies for their support.

Open Access This article is distributed under the terms of the Creative Commons Attribution Noncommercial License which permits any noncommercial use, distribution, and reproduction in any medium, provided the original author(s) and source are credited. 


\section{References}

1. A. Bolshakova et al., Eur. Phys. J. C 62, 293 (2009). CERN-PHEP-2008-022, arXiv:0901.3648

2. A. Bolshakova et al., Eur. Phys. J. C 62, 697 (2009). CERN-PHEP-2008-025, arXiv:0903.2145

3. A. Bolshakova et al., Eur. Phys. J. C 63, 549 (2009). CERN-PHEP-2009-009, arXiv:0906.0471

4. A. Bolshakova et al., Eur. Phys. J. C 64, 181 (2009). CERN-PHEP-2009-012, arXiv:0906.3653

5. A. Bolshakova et al., Eur. Phys. J. C 66, 57 (2010). CERN-PHEP-2009-025, arXiv:0912.0378

6. A. Bolshakova et al., Eur. Phys. J. C 56, 323 (2008). CERN-PHEP-2008-007, arXiv:0804.3013

7. S. Agostinelli et al., Nucl. Instrum. Methods Phys. Res. A 506, 250 (2003)

8. J. Allison et al., IEEE Trans. Nucl. Sci. 53, 270 (2006)

9. V. Uzhinsky et al., Eur. Phys. J. C 61, 237 (2009)

10. A. Ferrari et al., CERN-2005-10 (INFN-TC-05-11, SLAC-R773), FLUKA: a multi-particle transport code

11. G. Battistoni et al., in Proc. of the Hadronic Shower Simulation Workshop, Fermilab (Batavia, Illinois), 6-8 September 2006, ed. by M. Albrow, R. Raja. AIP Conf. Proc., vol. 896 (2007), p. 31. The FLUKA code: Description and benchmarking
12. V. Ammosov et al., Nucl. Instrum. Methods Phys. Res. A 588, 294 (2008). CERN-PH-EP-2007-030

13. V. Ammosov et al., Nucl. Instrum. Methods Phys. Res. A 578, 119 (2007). CERN-PH-EP-2007-005

14. A. Bolshakova et al., CERN-HARP-CDP-2009-001

15. A. Bolshakova et al., CERN-HARP-CDP-2009-002

16. A. Bolshakova et al., CERN-HARP-CDP-2009-003

17. A. Bolshakova et al., CERN-HARP-CDP-2009-004

18. A. Bolshakova et al., CERN-HARP-CDP-2009-005

19. J. Apostolakis et al., Progress in hadronic physics modelling in Geant4. J. Phys. Conf. Ser. 160, 012073 (2009)

20. M.G. Catanesi et al., Phys. Rev. C 77, 055207 (2008). arXiv:0805.2871

21. M. Apollonio et al., Phys. Rev. C 80, 065207 (2009). CERN-PHEP-2009-021, arXiv:0907.1428

22. N.V. Mokhov, Report Fermilab-FN-628 (1995)

23. N.V. Mokhov, S.I. Striganov, in Proc. of the Hadronic Shower Simulation Workshop, Fermilab (Batavia, Illinois), 6-8 September 2006, ed. by M. Albrow, R. Raja. AIP Conf. Proc., vol. 896 (2007), p. 50

24. V. Ammosov et al., J. Instrum. 3, P01002 (2008)

25. V. Ammosov et al., Eur. Phys. J. C 54, 169 (2008)

26. V. Ammosov et al., CERN-HARP-CDP-2006-003

27. V. Ammosov et al., CERN-HARP-CDP-2006-007

28. V. Ammosov et al., CERN-HARP-CDP-2007-001 